This item was submitted to Loughborough's Research Repository by the author.

Items in Figshare are protected by copyright, with all rights reserved, unless otherwise indicated.

\title{
GPU implementation of DPSO-RE algorithm for parameters identification of surface PMSM considering VSI nonlinearity
}

PLEASE CITE THE PUBLISHED VERSION

http://dx.doi.org/10.1109/JESTPE.2017.2690688

PUBLISHER

(C) IEEE

VERSION

AM (Accepted Manuscript)

LICENCE

CC BY-NC-ND 4.0

\section{REPOSITORY RECORD}

Liu, Zhao-Hua, Hua-Liang Wei, Qing-Chang Zhong, Kan Liu, and Xiao-Hua Li. 2019. "GPU Implementation of DPSO-RE Algorithm for Parameters Identification of Surface PMSM Considering VSI Nonlinearity”. figshare. https://hdl.handle.net/2134/25085. 


\title{
GPU Implementation of DPSO-RE Algorithm for Parameters Identification of Surface PMSM Considering VSI Nonlinearity
}

\author{
Zhao-Hua Liu, Member, IEEE, Hua-Liang Wei, Qing-Chang Zhong, Fellow, IEEE, Kan Liu ,Member, IEEE, and \\ Xiao-Hua Li
}

\begin{abstract}
In this study, an accurate parameter estimation model of surface permanent magnet synchronous machines (SPMSM) is established by taking into account voltage-source-inverter (VSI) nonlinearity. A fast dynamic particle swarm optimization (DPSO) algorithm combined with a receptor editing (RE) strategy is proposed to explore the optimal values of parameter estimations. This combination provides an accelerated implementation on graphics processing unit (GPU), and the proposed method is therefore referred to as G-DPSO-RE. In G-DPSO-RE, a dynamic labor division strategy is incorporated into the swarms according to the designed evolutionary factor during the evolution process. Two novel modifications of the movement equation are designed to update the velocity of particles. Moreover, a chaotic-logistic based immune receptor editing operator is developed to facilitate the global best individual (gBest particle) to explore a potentially better region. Furthermore, a GPU parallel acceleration technique is utilized to speed up parameter estimation procedure. It has been demonstrated that the proposed method is effective for simultaneous estimation of the PMSM parameters and the disturbance voltage $\left(V_{\text {dead }}\right)$ due to VSI nonlinearity from experimental data for currents and rotor speed measured with inexpensive equipment. The influence of the VSI nonlinearity on the accuracy of parameter estimation is analyzed.
\end{abstract}

Index Terms: particle swarm optimization (PSO), artificial immune system (AIS), Graphics Processing Unit (GPU), parallel computing, parameter estimation, permanent magnet synchronous machines (PMSMs), voltage-source-inverter (VSI), nonlinearity.

\section{I . INTRODUCTION ${ }^{1}$}

$\mathrm{R}$ ReCEnTly, permanent magnet synchronous machines (PMSMs) are widely used in high-performance applications such as industrial robots, servo drive, wind power generation, and machine tools due to their overall good performance

\footnotetext{
${ }^{1}$ Manuscript received November 1, 2016; revised January 3, 2017, and February24, 2017; accepted March 22, 2017. This work was supported in part by the National Natural Science Foundation of China under Grant (61503134, 61573299, 51374107, 51577057), the Hunan Provincial Education Department outstanding youth project under Grant (15B087), and the China Postdoctoral Science Foundation funded project under Grant (2013M540628, 2014T70767).

Z.-H. Liu and X.-H. Li are with the School of Information and Electrical Engineering, Hunan University of Science and Technology, Xiangtan, 411201, China (e-mail:zhaohualiu2009@hotmail.com, lixiaohua_0227@163.com).

H.-L. Wei is with the Department of Automatic Control and Systems Engineering, The University of Sheffield, Sheffield S1 3JD, U.K(w.hualiang@sheffield.ac.uk). Q.-C. Zhong is with Department of Electrical and Computer Engineering, Illinois Institute of Technology, $10 \mathrm{~W}$ 35th Street, IIT Tower 16C6-2, Chicago, IL, USA. (email:zhongqc@iee.org).

K.Liu is with the Control Systems Research Group, Loughborough University, Loughborough LE11 3TU, U.K (email: k.liu3@ lboro.ac.uk)
}

[1]-[2]. The design of the control system of such a machines crucially involves the choice of many key machine parameters such as winding resistance, $d q$-axis inductances, and rotor PM flux linkage [3][4]. Any change of these parameters could affect the system behavior and therefore the change in these parameters can be used to evaluate the health conditions of PMSM. For example, the inter-turn short circuit can result in an abrupt change in winding resistance and inductance [5]; the demagnetization can result in a sudden decrease in the amplitude of fundamental back electromotive force (EMF) [6]. A controller with inappropriately designed parameters cannot work well, and can lead to a dysfunction of the machine especially for high power machines and large scale equipment or systems [4]. A PMSM is time-varying system, whose parameters are sensitive to the change of environmental conditions such as temperature, noise, load torque, and the aging of the motor, etc. [7].

Recently, many parameter estimation approaches have been introduced in the literatures. Some measurement instruments such as thermal couplers, search coils and load test bench [8] were applied to observe the machine parameters. However, they are usually not preferred in practice for the potential increase of cost. In practical engineering, parameter optimization using numerical methods is an ideal technology for directly estimating the needed parameters based on regular measurable data [7]. Existing research mainly focused on online estimation algorithms including self-commissioning technique [2] ,extended Kalman filter (EKF) [9], model reference adaptive system (MRAS) [10], recursive least-squares (RLS) [3] [11], adaptive observer [12], and artificial neural networks (ANN) [10] [11]. However, with the increasing complexity of operation conditions, these methods may not always work well. For example, in [9], an EKF algorithm was proposed to estimate the rotor speed and position of PMSM, but it may be difficult for real applications as the algorithm is sensitive to noise. The MRAS estimators proposed in [10] cannot simultaneously estimate winding resistance, inductance and rotor flux linkage accurately. In this method, in order to estimate a group of parameters, some are fixed to their nominal values for the estimation of other parameters given in the motor manual. For example, the winding resistance parameter needs to be set to its nominal value in order to estimate the rotor flux linkage afterward, the accuracy of estimation results depend on the accuracy of the nominal values of machine. However, the nominal value is usually not consistent with the actual operating value, thus these 
estimators cannot ensure to converge to the actual parameter value. In comparison with other algorithms, RLS possesses a good property of rapid convergence rate, but the algorithm may suffer from high computational burden and poor tracking ability in non-stationary environments [3].An adaptive observer was introduced in [12] to obtain the estimated values of PMSM systems. It can estimate the PMSM parameters accurately, but has poor robustness when dealing with the uncertainties in machine parameters estimation. Usually, the existing parameter estimation models are based on the conventional $d q$-axis equation, the $d q$-axis equations will be rank deficient for estimating three or four parameters when the motor is operating at a steady state, thus the estimation results may converge to suboptimal. To solve the rank deficient problem, the $d$-axis current injection method can be used to increase the number of state equations due to the variation of $d$-axis current [11]. PMSM is usually fed by a voltage source inverter (VSI), and the reference voltages for the parameter estimator are measured from the PI regulator in a PMSM vector control system. This may introduce an error between the reference voltage of the controller and the actual output voltage of the VSI. Commonly, the researcher use the relevant parameters of the switching devices to calculated the VSI disturbance voltage, and then to compensate the reference voltage of the controller [13].However, some device parameters (e.g. the dead-time period, switching times and voltage drops of switching device) vary with the operating conditions and they difficult to be measured by instruments. Thus, the parameter estimates could be biased due to the effect of nonlinearity of VSI such as switch voltage drop, switching delay and dead zone response [14]. To achieve accurate estimation, the influence of VSI nonlinearity has to be considered; in other words, it is necessary to take into consideration of both the VSI and machine. Additionally, the parameters of the PMSM system are inherently dependent on each other, and this is a big challenge for most conventional parameter estimation methods.

More recently, a particle swarm optimization (PSO) algorithm was introduced for the estimation of parameter electrical machines including induction machine and PMSM machine [15]-[20]. The PSO algorithm is a nature-inspired algorithm with several advantages such as its self-tuning decoupling and fast convergence speed in dealing with multivariate coupling system parameter optimization problems [15]. A PSO combing least mean squares (LMS) method was proposed to identify the parameters of an induction motor in [16]. In [18], a co-evolution based parameter estimator was developed to estimate the multi-parameters of PMSM by combining multiple PSO and artificial immune system (AIS). However, the computational load of this method is heavy. A parallel implementation of co-evolutionary immune PSO on GPU is proposed to accelerate the computation of parameter estimation and temperature monitoring in PMSM [19], for which the estimation accuracy and time consuming of the parameter estimates were greatly improved by combining PSO with a parallel computing technology. Nevertheless, the existing PSO-based parameter estimators of PMSM are based on the basic $d q$-axis equation and do not consider the VSI nonlinearity. A dynamic PSO embed with variable exploration vector and Gaussian-distribution based dynamic opposition-based learning operator is proposed for the estimation of machine parameters and voltage-source-inverter (VSI) nonlinearities in PMSM [20]. The development of a high performance PSO for the estimation of PMSM multi-parameters, together with the VSI nonlinearity is still highly demanded.

In order to achieve better estimates for PMSM parameters, two important issues need to be solved when applying PSO algorithms. Firstly, the dynamic performance of the PSO need to be improved as the swarms are easily clustering together and losing their diversity in the later stage of evolution. Secondly, PSO would be time demanding if a large population size involves in the evolution and therefore some massively parallel devices may be required to accelerate the calculating speed. To overcome these problems, the labor division and cooperation mechanism ubiquitously existing in the biological world, together with the immune receptor editing mechanism in $A I S$, can be used to improve the dynamic performance of PSO during the search process. The time consuming problem can be solved by using Graphic processing units (GPUs), due to its massively parallel computing ability with hundreds of threads and low hardware cost [21].

This study aims to achieve better performance in PMSM parameters estimation using an accurate parameter estimation model where the effect of VSI nonlinearity is considered. A fast dynamic particles swarm optimization algorithm using immune receptor editing combined GPU acceleration technology for PMSM parameter optimization (called G-DPSO-RE) is proposed. The main contributions and main advantages can be summarized as follows:

1) An accurate parameter estimation model of surface permanent magnet synchronous machines (SPMSM) is established by taking into account voltage-source-inverter (VSI) nonlinearity. A labor division based dynamic particle swarm optimization (DPSO) algorithm combined with a receptor editing (RE) strategy is designed to explore the optimal values of parameter estimator.

2) High-performance computing ability of GPU is fully utilized to speed up parameter estimation procedure. It can promote the practical application and real-time response of PSO as it takes full advantage of the inherent parallelism of population-based intelligent computing techniques.

The remainder of this paper is organized as follows. In section II, an accurate parameter estimation model is established. In section III, the G-DPSO-RE algorithm for PMSM parameter estimation is proposed, where the principle, mathematical model and implementation procedure of the algorithm are addressed in details. Experimental results and analysis are given in section IV. Finally, conclusions are summarized in section $\mathrm{V}$.

\section{II . PMSM MODEL AND DESIGN OF PARAMETER ESTIMATION MODEL}

\section{A. PMSM Model}

The mathematical model of the PMSM in $d q$-axis voltage 
equation is given as

$$
\left\{\begin{array}{l}
u_{d}=R i_{d}+L_{d} \frac{d i_{d}}{d t}-L_{q} \omega i_{q} \\
u_{q}=R i_{q}+L_{q} \frac{d i_{q}}{d t}+L_{d} \omega i_{d}+\psi_{m} \omega
\end{array}\right.
$$

where $\omega$ is the electrical angular velocity, $u_{d}, u_{q}, i_{d}$ and $i_{q}$, are $d q$ axis stator voltage and current. The elements of the parameter $\operatorname{set}\left\{R, L_{d}, L_{q}, \psi_{m}\right\}$ are the motor winding resistance, magnet flux, $d$-axis and $q$-axis inductances, respectively, which are usually unknown to the users. Note that the estimated resistance $R$, as a lumped circuit resistance, includes two parts, namely, the ON-state slope resistances of the active switch and freewheeling diode in inverter and terminal wire resistance. At steady state the equation (1) can be discretized as follow.

$$
\left\{\begin{array}{l}
u_{d}(k)=R i_{d}(k)-L_{q} \omega(k) i_{q}(k) \\
u_{q}(k)=R i_{q}(k)+L_{d} \omega(k) i_{d}(k)+\psi \omega(k)
\end{array}\right.
$$

In a PMSM vector control system, the voltages used for the PMSM parameter estimation are usually measured from the output voltage of the current controllers, and the terminal voltages of PMSM are PWM pulses from VSI which are difficult to measure directly [22]-[24]. The two output voltages, denoted by $u_{d}{ }^{*}, u_{q}{ }^{*}$, are shown in Fig.1. Note that there exists an error between the reference voltage of the controller and the actual output voltage of the VSI due to the nonlinearity of VSI, so it is essential to estimate VSI nonlinearity.

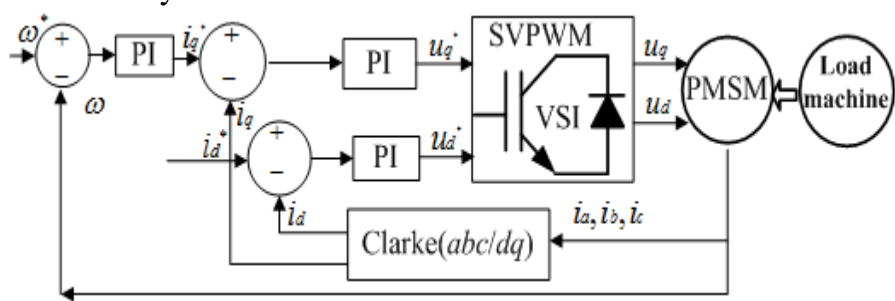

Fig.1.The schematic of vector controlled PMSM drive system.

Taking into account the influence of VSI nonlinearity, the model of PMSM and VSI as a whole, for surface-mounted PMSM, $d$-axis inductance is regarded as equal to $q$-axis inductance, that is $L_{\mathrm{d}}=L_{\mathrm{q}}=L$, (2) can be rewritten as

$$
\begin{aligned}
& u_{d}{ }^{*}(k)+D d(k) V_{\text {dead }}=R i_{d}(k)-L \omega(k) i_{q}(k) \\
& u_{q}{ }^{*}(k)+D q(k) V_{\text {dead }}=R i_{q}(k)+L \omega(k) i_{d}(k)+\psi_{m} \omega(k)
\end{aligned}
$$

where $D_{d}$ and $D_{q}$ are the function of rotor position[13] , in (3) $L, R, \psi_{m}$ and $V_{\text {dead }}$ are the parameters to be estimated. The variable $V_{\text {dead }}$ is the distorted voltage due to VSI nonlinearity, and can be represented as

$$
V_{\text {dead }}=\frac{T_{d e a d}+T_{o n}-T_{o f f}}{T s} \cdot\left(V_{d c}-V_{s a t}+V_{d}\right)+\frac{V_{s a t}+V_{d}}{2}
$$

where $T_{\text {dead }}$ is the dead-time period of the switching device, $T_{\text {on }}$ and $T_{\text {off }}$ are turn-on and turn-off times of the switching device, $V_{d c}$ is measured real-time dc bus voltages, $V_{\text {sat }}$ and $V_{d}$ are the saturation voltage drop of the active switch and the forward voltage drop of the freewheeling diode, $T_{\mathrm{s}}$ is the switching period. It can be seen that if variable $V_{\text {dead }}$ is ignored, the estimation results may also be influenced by the nonzero VSI nonlinearity terms (Dd. $V_{\text {dead }}$ and $D q . V_{\text {dead }}$ ), and this may introduce an error into the estimation of the PMSM parameters.

\section{B. The Design of Estimation Model based on Parameter Optimization}

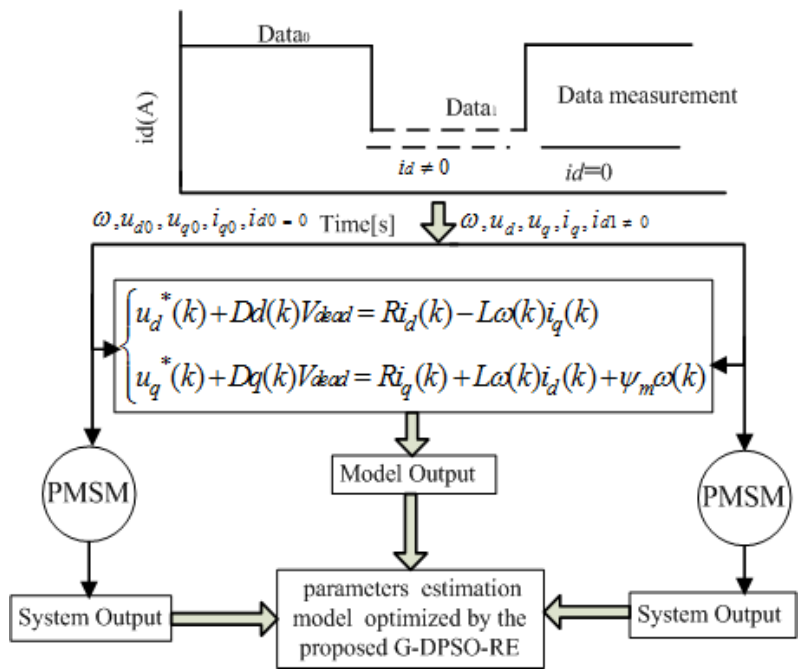

Fig .2.Schematic diagrams of estimation and mathematical model.

Apparently, the rank of equation $(3)<3$, while the number of unknown parameters is four, thus the equation (3) is rank deficient, the four parameters in (3) are not be identifiable and an estimate to converge to suboptimal. To solve this problem, a full rank reference model should be constructed if all these parameters need to be estimated simultaneously at steady state. Generally, $d$-axis current injection method is employed to obtain more state equations due to the variation of $d$-axis current .The parameters of machine can be assumed to be constant as the duration of injected pulse current is very short due to mechanical inertia and fast response of current loop PI controller. In this case, the influence of injecting a short pulse of $i_{d}$ on output torque and speed can be negligible. Thus, the two sets of steady state data (Data0 and Data1) can be used together for the estimation machine parameters and VSI nonlinearity simultaneously modeling. An illustration is given in Fig.2, where $i_{d 0}=0$ (A) during normal operation for the decoupling the flux and torque control of SPMSM, and a very short time of $i_{d l} \neq 0$ (A) is injected to obtain another $d q$-axis voltage equation model. Two groups of equations at $i_{\mathrm{d}}=i_{\mathrm{d} 0}$ and $i_{\mathrm{d}}=i_{\mathrm{d} 1}$ are obtained as

$$
\left\{\begin{array}{l}
u_{d 0}(k)=-L \omega(k) i_{q 0}(k)-D d 0(k) V_{\text {dead }} \\
u_{q 0}(k)=R i_{q 0}(k)+\psi_{m} \omega(k)-D q_{0}(k) V_{\text {dead }} \\
u_{d 1}(k)=R i_{d 1}(k)-L \omega(k) i_{q 1}(k)-D d_{1}(k) V_{\text {dead }} \\
u_{q 1}(k)=R i_{q 1}(k)+L \omega(k) i_{d 1}(k)+\psi_{m} \omega(k)-D q_{1}(k) V_{\text {dead }}
\end{array}\right.
$$


The parameter identification can be addressed as an optimization problem where the system response to a known input is used to find the unknown parameter values of a model. Based on (5), the cost function for the estimating parameter set $\left(R, L, \psi_{m}, V_{\text {dead }}\right)$ is as

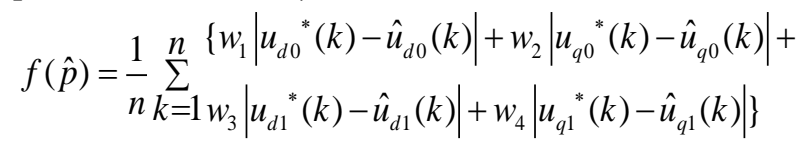

where $w_{1}, w_{2}, w_{3}, w_{4}$ are weight coefficients, satisfying $w_{1}=w_{2}=w_{3}=w_{4}=1 . \quad \hat{u}_{d}$, and $\hat{u}_{q}$ indicate the estimated voltages in $d q$-axis computed by the measured currents and the estimated parameters.

\section{GPU-ACCELERATED PARALLEL DYNAMIC PSO WITH RECEPTOR EDITING}

\section{A. Principle of Basic PSO Algorithm}

PSO [15] is a swarm-based intelligent optimization algorithm inspired by the ideas of simulating behaviors of bird flocking foraging. Assuming that each particle $i$ in a $d$-dimensional solution space is composed of two vectors, which are the velocity vector $V_{i}=\left\{V_{i 1}, V_{i 2, \ldots,}, V_{i d}\right\}$ and the position vector $X_{i}=\left\{X_{i 1}, X_{i 2}, \ldots, X_{i d}\right\}$, the search procedure can be formulated as

$$
\begin{aligned}
V_{i d}(t+1)= & \phi V_{i d}+c_{1} * \operatorname{rand}_{1}()\left(\text { Pbest }_{i d}(t)-X_{i d}(t)\right) \\
& +c_{2} * \operatorname{rand}_{2}()\left(\text { gBest }_{d}(t)-X_{i d}(t)\right) \\
& X_{i d}(t+1)=X_{i d}(t)+V_{i d}(t+1)
\end{aligned}
$$

where $c_{1}$ and $c_{2}$ are the acceleration coefficients, $\phi$ is the inertia weight factor decreasing linearly, rand $_{1}$ and rand $_{2}$ are random numbers in the interval $[0,1]$, respectively. Pbest $t_{i d}$ represents the best position with the best fitness found by $i$-th particle up to now and gBest $_{d}$ is the best position found among the entire population.

\section{B. The proposed G-DPSO-RE Algorithm for PMSM parameter estimation}

As mentioned in Section II, the objective function is multimodal and therefore requires that the optimization method should have a good global search capability. The existing static optimization methods may easily get trapped in some local minima. To effectively solve the multimodal optimization problem (6), a fast dynamic parameter tracking approach is indeed developed to explore the optimal search ability for parameters estimator of the PMSM. Biological inspired PSO, combined with parallel computing technology, can meet such a requirement, since PSO has the intrinsic ability to automatically track the dynamic objective and the GPU acceleration technology can reduce the computation time with significantly low cost. The proposed G-DPSO-RE method involves three key strategies.

1) Firstly, three novel schemes are developed to enhance the dynamic performance of PSO based on a division of labor concept in colony society. One of the designs is to divide the group into two parts dynamically according to evolution factor during the evolution process and two novel velocity updating equations are investigated for two different state particles respectively.

2) Secondly, a novel strategy is to utilize $R E$ using chaotic logistic to overcome the blindness in action of gBest particles stochastic evolution and make it drift from the local minima. 3) Thirdly, GPU parallel computing technique is used to speed up the search process and then an optimized parallel accelerated G-DPSO-RE algorithm using CUDA (Compute Unified Device Architecture, is a GPU programming hardware and software architecture developed by NVIDIA Corporation).

The general steps of G-DPSO-RE for PMSM parameter estimation are stated as follows.

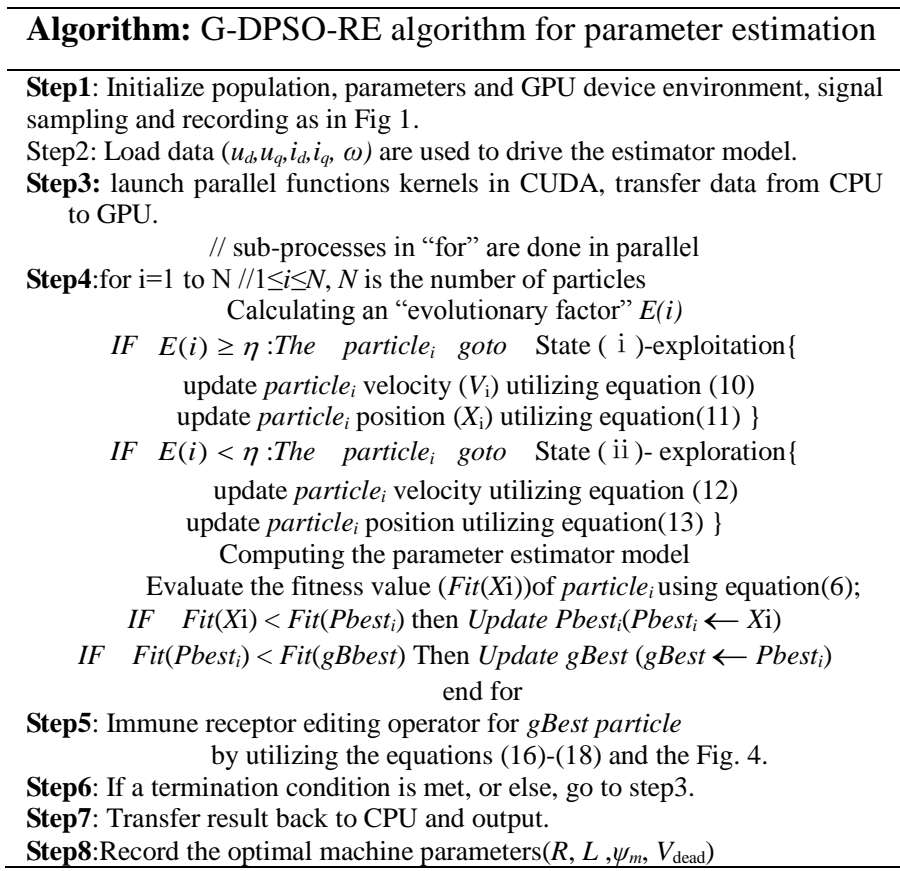

\section{Dynamic PSO model}

In PSO, each particle of swarm moves in a random direction, it has a potential trend of clustering together and may lose its diversity in the later stage of evolution. In a colony society, in order to get rich food, swarms perform different tasks simultaneously via collaborating with each other among their individual members (particles); some particles play a role for predation, and for food exploration. This is called a labor division. Based on the idea of the division of labor in nature life systems [25] [26], the group is divided into two different subgroups including exploitation group and exploration group during the evolution process according to the evolutionary state. A number of 'good' particles should be able to refine their search performance step by step, converge to the best-known locations rapidly and then carry out a better search in the next step. Other particles should get larger momentum, and be able to jump out from local points and explore better search regions. Following the idea of natural evolution, the entire population is decomposed into two sates as shown in Fig. 3. In this model, particles are divided into two categories: 1) exploitation state and 2) exploration state. 


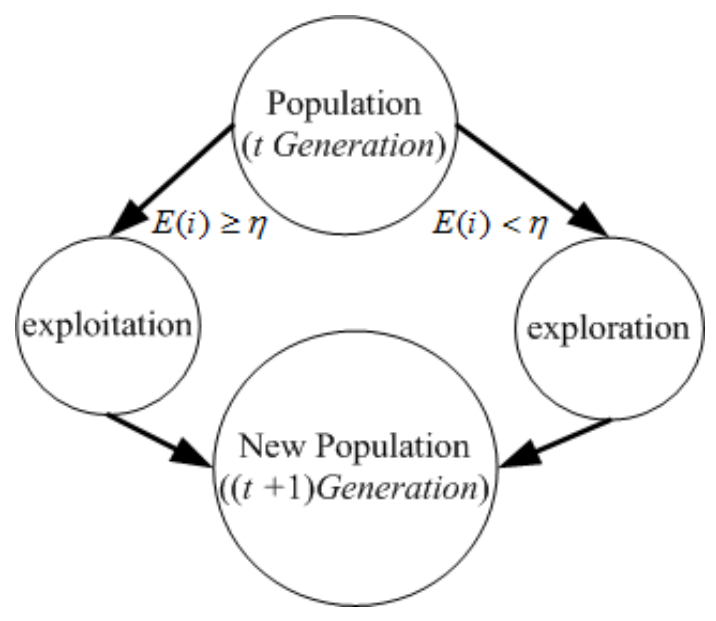

Fig.3. Dynamic evolution model for PSO

In order to achieve the automatic control of population dynamic division, a real-time evolutionary state estimation procedure is performed to identify the two evolutionary states via computing an "evolutionary factor" of each individual. Therefore, in this mechanism the population evolutionary information in every generation has been taken into account, and details are given below.

1) Denote the best fitness of the $i$-th particle at the $t$-th generation by $\mathrm{Fit}^{i}{ }_{\text {best }}$, compute an "evolutionary factor" $E(i)$.

$$
E(i)=\frac{\text { Fit }^{i}{ }_{\text {best }}(t+1)-\text { Fit }^{i}{ }_{\text {best }}(t)}{\text { Fit }^{i}{ }_{\text {best }}(t)-\text { Fit }^{i}{ }_{\text {best }}(t-1)+\alpha}
$$

where $t$ is current generation, $T$ is the total generation, $\alpha$ is a smooth coefficient. Let $\eta=e^{-t / T}$ be a function measuring the convergence rate of the evolutionary factor $E(i)$. If $E(i) \geq \eta$, then the particle $i$ should converge to the exploitation state and carry out a fine search. Otherwise, if $E(i)<\eta$, then the particle $i$ should shift to the exploration state and make a broader exploration of the solution space. Therefore, the designed dynamic evolution model makes particles more flexible in exploration and exploitation and is suitable to solve dynamic problems. Thus, the size of each subpopulation can be dynamically adjusted based on individual's evolutionary status.

2) State ( i )-exploitation: In this exploitation state, we use the following velocity updating equation

$$
\begin{gathered}
V_{i d}(t+1)=\phi V_{i d}+c_{1} * \operatorname{rand}_{1}()\left(\text { Pbest }_{\varphi[d]}(t)-X_{i d}(t)\right) \\
+c_{2} * \operatorname{rand}_{2}()\left(\text { gBest }_{d}(t)-X_{i d}(t)\right) \\
X_{i d}(t+1)=X_{i d}(t)+V_{i d}(t+1)
\end{gathered}
$$

where $g B e s t 1$ is the best position discovered in the entire particles under exploitation state, the symbol $\varphi$ is the randomly selected the exploitation population and $\varphi=\lfloor$ rand $* K\rfloor, k$ is total exploitation population size. The velocity updating equation of exploitation state indicates that all of exploitation particles' historical best information is used to update a particle's velocity. So, the elite particles in exploitation state can focus on the best-known solution region and search the optimal goal via the cooperative behavior of the entire sub-swarms.

3) State (ii ) - exploration: In this exploitation state, we use the following velocity updating equation:

$$
\begin{aligned}
& V_{j d}(t+1)=c_{1} * \text { rand }_{1}()\left(\text { Pbest }_{j d}(t)-X_{j d}(t)\right) \\
& +c_{2} * \text { rand }_{2}()\left(\text { gBest }_{d}(t)-X_{j d}(t)\right) \\
& +\left(X_{\max }-X_{\min }\right) \cdot \text { Gauss } \\
& \quad X_{i d}(t+1)=X_{i d}(t)+V_{i d}(t+1)
\end{aligned}
$$

where $g$ Best 2 is the best position discovered in the entire particles under exploration state. In this velocity updating equation, the old velocity $\phi V_{i d}$ component is omitted, that means the potential local information is forgotten .And also, the term $(X \max -X \min )$.Gauss is added to provide a broader exploration of the solution space for the $j$-th particle. The symbol Gauss is the density function with a zero mean $u$ and a standard deviation (SD) $\sigma$, which can be expressed as:

$$
\begin{gathered}
\operatorname{Gauss}(x)=\frac{1}{\sigma \sqrt{2 \pi}} \exp \left(-\frac{(x-u)^{2}}{2 \sigma^{2}}\right) \\
\sigma=\sigma_{\max }-\left(\sigma_{\text {max }}-\sigma_{\text {min }}\right) \frac{t}{T}
\end{gathered}
$$

where $\sigma_{\max }$ and $\sigma_{\min }$ are the upper and lower bounds of $\sigma(\mathrm{in}$ this paper fixed $\sigma_{\max }=1, \sigma_{\min }=0.01$ ). From the above discussion, it can be expected that the dynamic PSO scheme can make equilibrium between extensive searching and accurate searching.

\section{Chaotic Logistic-Based Receptor Editing for gBest}

The gBest particles usually used as the exemplars to lead the flying direction of other particles among the swarms. It needs a reinforcement learning mechanism to improve the gBest search performance. It was discovered that B-lymphocytes in natural immune system with low quality will undergo a molecular selection and develop completely new ones by gene recombination or shift, which called immune receptor editing theory[27].The receptor editing mechanism can provide wider exploration of the solution space and help gBest push itself out to a potentially better region in unknown environment. If there another better region is found, then the rest of the swarm will follow the leader to jump out and converge to the better region. Nonlinear chaotic logistic series possesses the characteristics of randomness, ergodicity and so on, which can simulate the operations of gene drift or recombination in immune receptor editing. The receptor editing operator is defined as:

$$
\begin{aligned}
& \text { gBest }^{d}=\text { gBest }^{d}+\left[\left(r d>P_{m}\right)\right] *\left(X_{\max }^{d}-X_{\min }^{d}\right) * x(t+1) \\
& -\left[\left(r d \leq P_{m}\right)\right] *\left(X_{\text {max }}^{d}-X_{\min }^{d}\right) * x(t+1)
\end{aligned}
$$




$$
\left[r d>P_{m}\right]=\left\{\begin{array}{l}
1 \ldots . \text { if } . . r d>P_{m} \\
0 \ldots \ldots \text { else }
\end{array}\right.
$$

Where $r d$ is a randomly generated number, and $P_{m}$ is set to be 0.5 , the search range $\left[x^{d}{ }_{\text {max }}, x_{\text {min }}^{d}\right]$ is the upper bound and lower bound respective. The term $x(t+1)$ is chaotic logistic sequence function:

$$
x(t+1)=u x(t)(1-x(t))
$$

where control parameters $u \in N$. The sequence (18) can exhibit chaotic behaviors when its initial values on $[0,1]$ except $0,0.25,0.50,0.75$, and 1.0 [28]. Thus, chaotic-logistic-based receptor editing can be viewed as a refinement mechanism, which can provides a broader exploration of the solution space for the gBest particle and can lead it to the global optimum.

\section{E. Parallelization Implementation on GPU}

Graphic processing unit (GPU) possesses an obvious advantage over CPU in terms of numerical processing ability, multithread instruction unit, and memory bandwidth, whereas it has a low cost and small power consumption, which can greatly reduce the required computing times. In order to speed up parameter estimation procedure, the proposed G-DPSO-RE algorithm is implemented in the GPU devices through the CUDA program. Both CPU and GPU are conducting heterogeneous collaborative computation where the GPU is carry out numeric parallel processing while CPU is in charge of serial computing such as logic and transaction processing.

Note that in the work the entire swarm is run at one block of GPU structure and each particle is run at one thread, as illustrated in Fig.4.The populations and related parameters are generated on CPU and allocated to a grid with one dimension of blocks at GPU. So, the proposed parameters estimation method can be speeded up significantly by GPU.

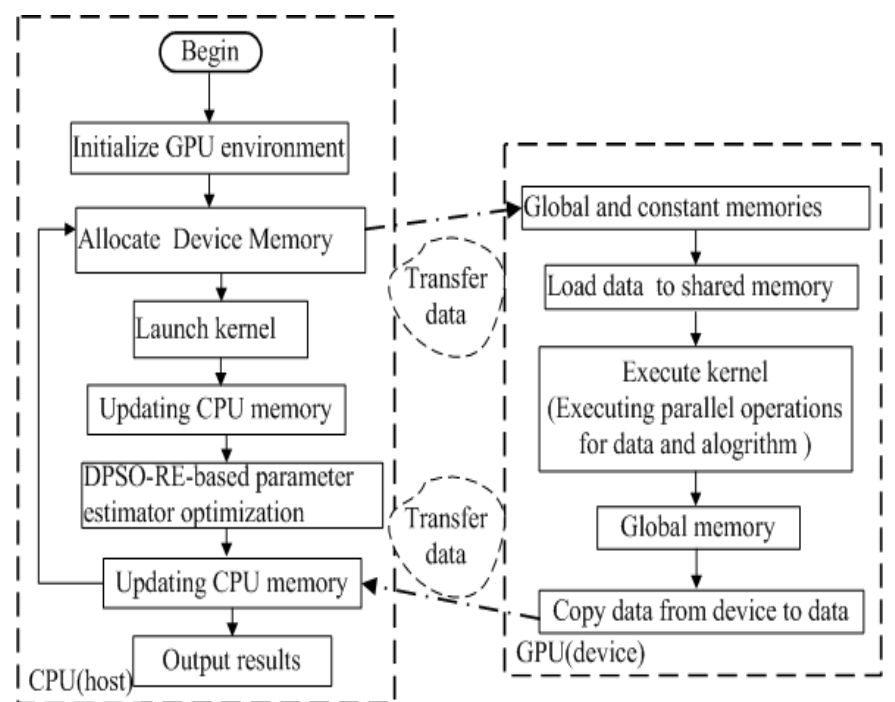

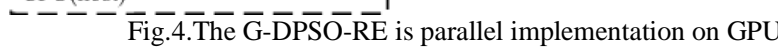

\section{EXPERIMENTAL RESULTS AND ANALYSIS}

A Hardware Control System and Software Platform
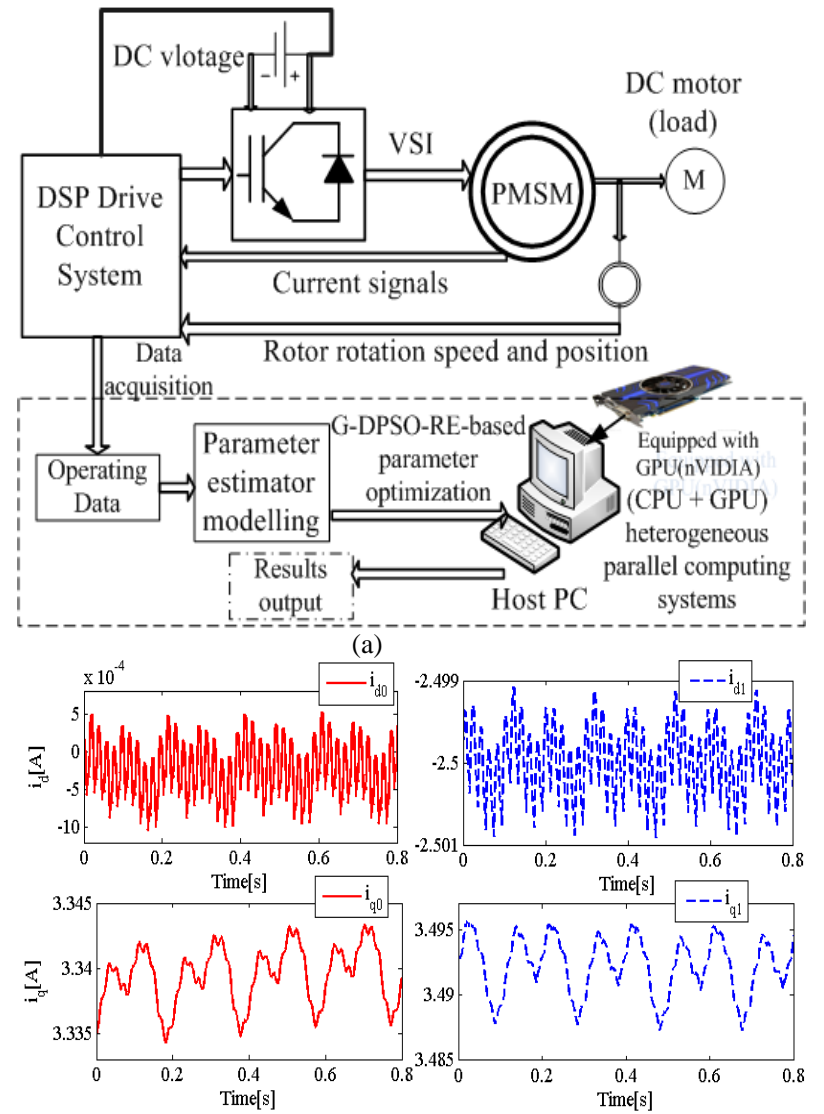

(b)
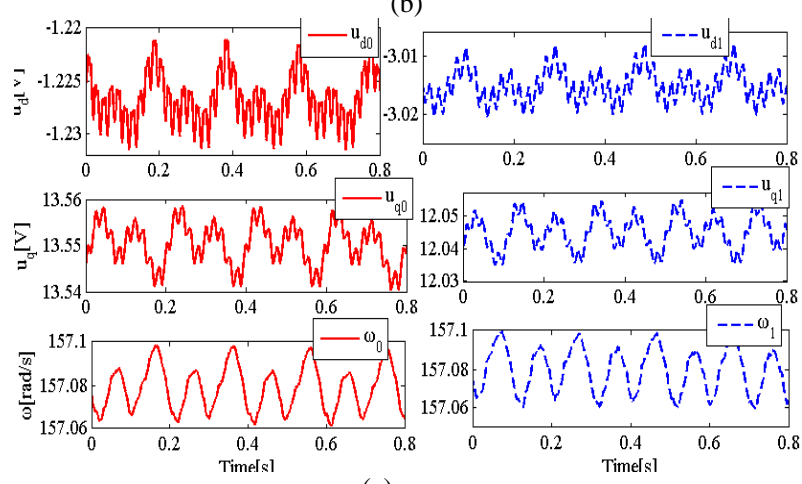

(c)

Fig. 5. Schematic diagram of identification system and waveforms of sampled operation data. (a) The schematic diagram hardware and software platform framework (b) Sampled of $d q$-axis current.(c) sampled of voltage and electrical angular speed .

TABLE I

DESIGN PARAMETERS AND SPECIFICATION OF PMSM

\begin{tabular}{cc}
\hline Rated speed & $400 \mathrm{rpm}$ \\
\hline Rated current & $4 \mathrm{~A}$ \\
\hline DC link voltage & $36 \mathrm{v}$ \\
Nominal terminal wire resistance & 0.043 \\
Nominal self inductance & $2.91 \mathrm{mh}$ \\
Nominal mutual inductance & $-0.330 \mathrm{mh}$ \\
Nominal $d$-axis inductance & $3.24 \mathrm{mh}$ \\
Nominal $q$-axis inductance & $3.24 \mathrm{mh}$ \\
Nominal amplitude of flux induced by & $77.6 \mathrm{mWb}$ \\
magnets & 5 \\
Number of pole pairs & $0.330 \Omega$ \\
Nominal phase resistance $\left(T=25{ }^{\circ} \mathrm{C}\right)$ & $0.8 \mathrm{e}-5 \mathrm{kgm}^{2}$ \\
Inertia
\end{tabular}


To perform our experiment, the schematic of the parameter estimation system is depicted in Fig.5(a), whose design parameters are shown in Table I, where a permanent magnet synchronous motor prototype and DSP vector control hardware platform are used as the experimental facility. The waveforms of measured $d q$-axis currents/voltages and electrical angular speeds of PMSM such as normal temperature condition are shown in Fig.5 (b)-(c). The current signals are obtained from the Hall transducers and then sampled by the DSP. The DC link is connected with the DC power source whose output is fixed to $36 \mathrm{~V}$. The sampling period is set to $83.3 \mu \mathrm{s}$. The data used for parameters estimation data modeling come from the DSP via serial protocol communication network and recorded in memory. After this, the parameter estimator is computed iteratively in host computer by the proposed G-DPSO-RE using visual studio 2012 software. For a large-scale engineering application, there is a need to process a large amount of operating condition data and control signals, so it needs large computing and mass storage, for such a case the processing of parameter estimation can still be done in a PC, equipped with graphic processing units (GPUs), which has massively parallel computing ability with hundreds of threads and low hardware cost. The work can be done by collaborative PC with inverter controller: the high computational task and massive storage can be done by PC and the results can be sent to inverter controller for controller design.

A number of hybrid PSOs are used for a comparison purpose with the proposed G-DPSO-RE, including HGAPSO (hybrid PSO with genetic algorithm) [29], HPSOWM (hybrid PSO with Wavelet Mutation) [30], CLPSO (comprehensive learning PSO) [31], A-CLPSO (An improved comprehensive learning PSO) [32] and APSO (adaptive Particle Swarm Optimization) [33]. To assess the performance of parameter estimation, a statistical analysis is performed in terms of the mean results, standard deviation and the t-test value. The basic settings of these PSOs are as follows: the maximum iteration is 300 and the number of runs is 15 . All hybrid PSOs are operated on the same platform with the same objective function and PMSM hardware. All experiments are carried out on the same computer with AMD Athlon(tm) II X4 555, four-core processors, RAM 4.0GB and GPU of NVIDIA GeForce GTX560TI equipped with 512 cores.

\section{B. Parameter Estimation under Normal Temperature Condition}

Table II presents the set of the parameters which are applied in the HGAPSO, HPSOWM, CLPSO, A-CLPSO, APSO, G-DPSO-RE algorithms for PMSM parameter estimation using data measured from normal temperature environment, and the convergence of different PSOs are shown in Fig.6 from which it is clear that the proposed G-DPSO-RE shows the best performance in terms of mean, standard deviations and $t$-values among those seven methods. Furthermore, all the $t$-values are higher than 9, which imply that the G-DPSO-RE has significantly better solution performance than other hybrid PSOs (the confidence level is 98\%). As can be seen from Fig.6, DPSO-RE converges to the optimum after about 60 generations of evolution whereas other hybrid PSOs shows poor convergence performance. Moreover, as shown in Table II , the execution time of G-DPSO-RE is shorter than the other seven methods.

As demonstrated in Table II and Fig.8 (a)-(c), the estimated winding resistance $(0.371 \Omega)$ by G-DPSO-RE is quite close to its measured value $(0.373 \Omega(0.33 \Omega+0.043 \Omega))$ under normal temperature condition. In addition, the estimated flux linkage $\psi_{m}(78.36 \mathrm{mWb})$ by G-DPSO-RE is quite close to its nominal value $(77.6 \mathrm{mWb})$, the estimated $d q$-axis inductance $(3.474 \mathrm{mH})$ is also consistent well with the nominal value on manual $(3.24 \mathrm{mH})$. As shown in Fig. 7, the value of VSI disturbance voltage $V_{\text {dead }}$ can be estimated along with other machine parameters based on the proposed estimator model. Although the accurate value of $V_{\text {dead }}$ cannot be acquired, it can be seen from Table II and III that the estimation results of the machine parameters (i.e. resistance (R), rotor flux linkage $\left(\psi_{m}\right)$ ) are of the highest estimation accuracy, and thus it can be expected that the estimated value of $\mathrm{V}_{\text {dead }}$ should be close to true value.

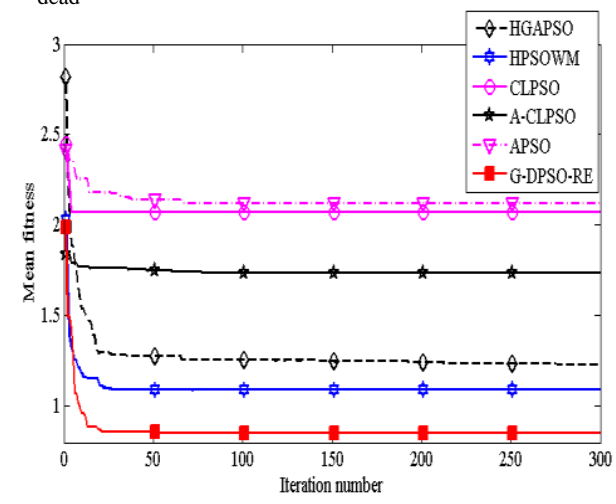

Fig. 6. The fitness convergence curve of six PSOs on PMSM parameter identification under normal temperature condition.

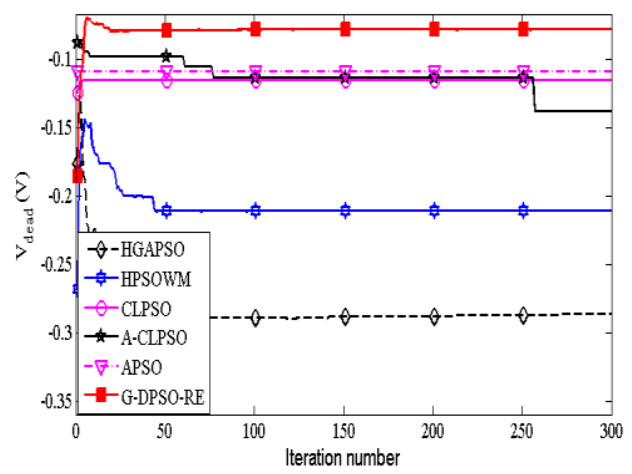

Fig.7.The estimated VSI distorted voltage under normal temperature condition In all, the proposed G-DPSO-RE is of high accuracy in parameter estimation under normal temperature condition though there is a slight difference between the estimated and nominal values of $\left(R, L, \psi_{m}\right)$ due to nonlinearity on load condition. It can also be observed from Table I and Fig.8(a)-(c) that the proposed G-DPSO-RE produced more precise parameter estimates for motor resistance, $d q$-axis inductances and the rotor flux, and the estimates converge to their desired values rapidly. The proposed estimator has a global convergence performance by combining the labor division cooperation mechanism inspired by the biological world and immune receptor editing mechanism in AIS. The 
results confirm that the cost function previously defined is related to the reality problems with local minima, and the poor performance of the identification method will progress from convergence to localization.

Moreover, as is shown in Table II, in terms of time-consuming, it requires $23.85 \mathrm{~s}, 36.24 \mathrm{~s}, 18.30 \mathrm{~s}, 18.17 \mathrm{~s}$, and $12.47 \mathrm{~s}$ for HGAPSO, HPSOWM, CLPSO, A-CLPSO and APSO, respectively .However, the computation time of G-DPSO-RE is only $6.97 \mathrm{~s}$, which is smaller than all the comparative PSO methods. All this demonstrates that the potential of the high-performance computing ability of GPU is well exploited to speed up the parameter estimation procedure. It can promote the real-time response of the proposed G-DPSO-RE as it takes the advantage of the inherent parallelism of population-based intelligent computing techniques.

As mentioned above, the proposed G-DPSO-RE for the estimation of PMSM parameters can converge to the global optimal solution when solving dynamically nonlinear PMSM parameters estimation problem.

TABLE II .

RESULT COMPARISONS AMONG SIX PSOS ON PMSM PARAMETER IDENTIFICATION WITH NORMAL TEMPERATURE

\begin{tabular}{|c|c|c|c|c|c|c|c|}
\hline \multicolumn{2}{|c|}{$\begin{array}{c}\text { Estimated } \\
\text { Parameters }\end{array}$} & HGAPSO & HPSOWM & CLPSO & A-CLPSO & APSO & G-DPSO-RE \\
\hline \multicolumn{2}{|c|}{$R(\Omega)$} & 0.359 & 0.367 & 0.333 & 0.321 & 0.369 & 0.371 \\
\hline \multicolumn{2}{|c|}{$\psi_{m}(m \mathrm{~Wb})$} & 78.23 & 77.69 & 78.62 & 78.51 & 79.62 & 78.36 \\
\hline \multicolumn{2}{|c|}{$L(m \mathrm{H})$} & 3.528 & 3.589 & 3.278 & 3.133 & 3.755 & 3.474 \\
\hline \multicolumn{2}{|c|}{$V_{\text {dead }}(\mathrm{V})$} & -0.286 & -0.211 & -0.116 & -0.139 & -0.109 & -0.078 \\
\hline \multirow{4}{*}{ Fitness } & Mean & 1.23 & 1.09 & 2.072 & 1.74 & 2.12 & 0.85 \\
\hline & Std.dev & 0.259 & 0.123 & 0.553 & 0.608 & 0.588 & 0.0045 \\
\hline & Time(s) & 23.85 & 36.24 & 18.30 & 18.17 & 12.47 & 6.97 \\
\hline & t-value & 10.04 & 12.11 & 15.51 & 10.29 & 15.17 & $\mathbf{0}$ \\
\hline
\end{tabular}

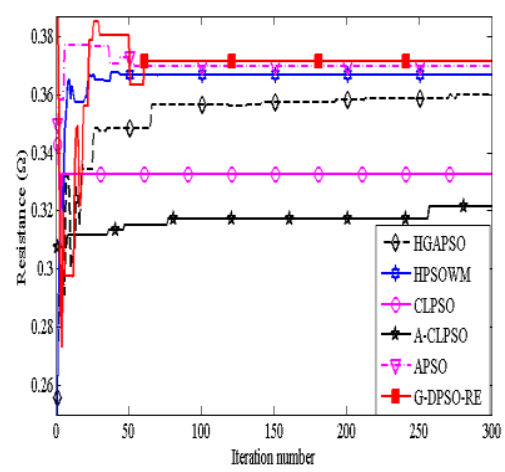

(a)

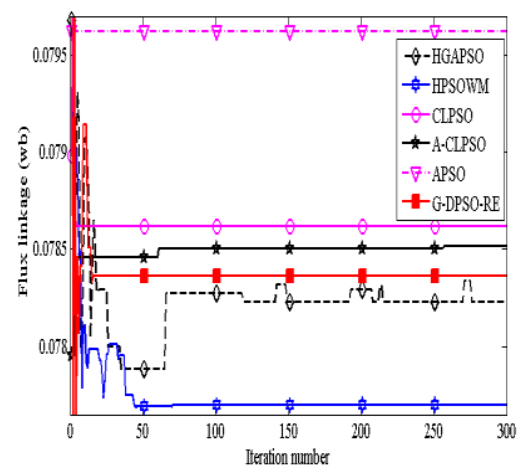

(b)

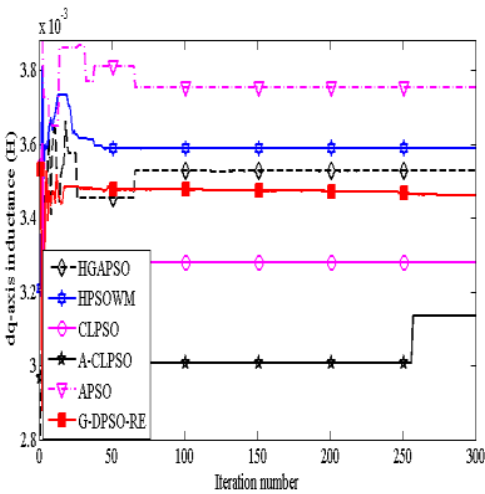

(c)

Fig. 8. Identified parameters (a) winding resistance. (b) rotor flux linkage (c) $d$-axis inductance.

\section{Parameter Estimation under Temperature Variation Condition}

Temperature is the main indicator for the reliable operation of PMSM, the variation of temperature can change the machine physical parameters. In order to evaluate the dynamic performance of the proposed method for tracking the change of parameters under temperature variation conditions, a heater is used to heat the prototype PMSM, and experiments are carried out. Firstly, continuously heating the PMSM for 20 minutes and then recording experimental data for the estimation of the machine parameters ( $t=20$ minutes).

The comparisons of the performance for different PSOs are shown in Table III , Fig.9. ,Fig.10.and Fig.11.The convergence curves of different PSOs are shown in Fig.9. From Table III, it is obvious that G-DPSO-RE produces the best performance in terms of mean, standard deviations and $t$-values. Fig.9 further shows that the G-DPSO-RE has a fast convergence speed compared to other hybrid PSOs.

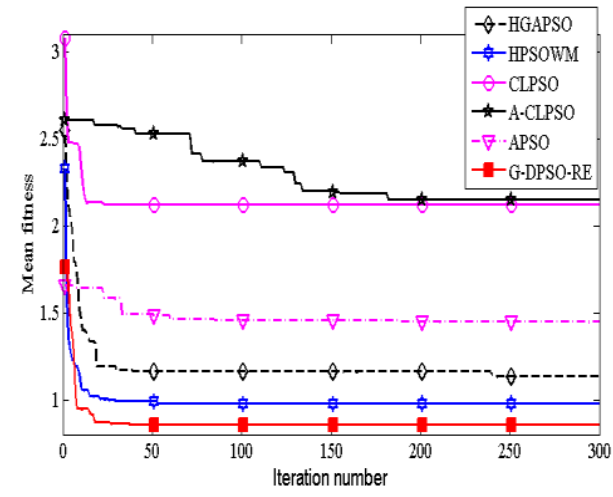

Fig. 9. The fitness convergence curve of six PSOs on PMSM parameter identification under variation temperature condition.

Additionally, the stability of the G-DPSO-RE is better than other hybrid PSOs. Meanwhile, as can be seen from Table III and Fig.11, the estimated winding resistance $R, d q$-axis inductance $L$ and rotor flux linkage $\psi_{m}$ vary with the changing temperatures. For example, the estimated winding resistance value varies from $0.371(\Omega)$ to $0.446(\Omega)$ after 20 minutes heating due to the effects of the thermal metal, the estimated 


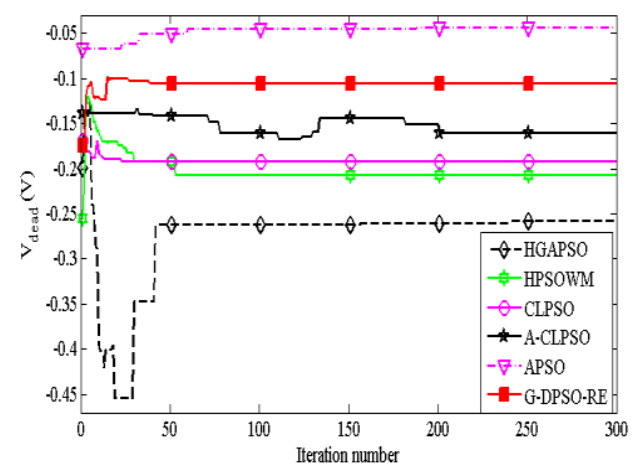

Fig.10.The estimated VSI distorted voltage under variation temperature condition

rotor flux linkage decreased from $78.36(\mathrm{mWb})$ to 76.81 $(m W b)$ after 20 minutes heating, the abrupt drop in the estimated rotor flux linkage after 20 minute heating can be explained by the fact that the residual flux density of the PM

TABLE III. reduces when the temperature of $\mathrm{NdFeB}$ magnets increases, since the flux density has changed during the data measurement after 20-minitue heating. The estimated VSI distorted voltage under variation temperature condition as in Fig.10. From Table II and Table III, it can be seen that the estimated VSI disturbance voltage $V_{\text {dead }}$ varies from $-0.078(\mathrm{~V})$ to $-0.107(\mathrm{~V})$ after 20 minute heating . This phenomenon can be explained by the fact that the VSI nonlinearity is also influenced by the temperature variation.

From above results and analysis, it indicates that G-DPSO-RE has a good dynamic tracking performance. Hence, the G-DPSO-RE is significantly better and statistically more robust than other listed hybrid PSOs in terms of global search capacity and local search precision in our experiments.

RESULT COMPARISONS AMONG SIX PSOS ON PMSM PARAMETER IDENTIFICATION UNDER TEMPERATURE VARIATION CONDITION

\begin{tabular}{|c|c|c|c|c|c|c|c|}
\hline \multicolumn{2}{|c|}{ Estimated Parameters } & HGAPSO & HPSOWM & CLPSO & A-CLPSO & APSO & G-DPSO-RE \\
\hline \multicolumn{2}{|c|}{$R(\Omega)$} & 0.478 & 0.457 & 0.479 & 0.419 & 0.473 & 0.446 \\
\hline \multicolumn{2}{|c|}{$\psi_{m}(m \mathrm{~Wb})$} & 75.84 & 76.08 & 74.98 & 77.00 & 75.14 & 76.81 \\
\hline \multicolumn{2}{|c|}{$L(m \mathrm{H})$} & 3.572 & 3.277 & 2.539 & 3.453 & 3.335 & 3.466 \\
\hline \multicolumn{2}{|c|}{$V_{\text {dead }}(\mathrm{V})$} & -0.258 & -0.207 & -0.602 & -0.161 & -0.044 & -0.107 \\
\hline \multirow{4}{*}{ Fitness } & Mean & 1.131 & 0.973 & 2.121 & 2.15 & 1.446 & 0.855 \\
\hline & Std.dev & 0.293 & 0.121 & 0.412 & 0.357 & 0.474 & 0.084 \\
\hline & Time(s) & 23.86 & 36.23 & 18.31 & 18.18 & 12.46 & 6.98 \\
\hline & t-value & 4.73 & 2.66 & 17.77 & 19.91 & 7.52 & 0 \\
\hline
\end{tabular}

TABLE IV

COMPUTING TIME FOR PMSM PARAMETER IDENTIFICATION BASED ON G-DPSO-RE WITH MULTI-CORE ARCHITECTURE

\begin{tabular}{|l|l|l|l|l|l|l|l|l|l|}
\hline \multicolumn{2}{|l|}{$\begin{array}{l}\text { CPU-with one } \\
\text { core }\end{array}$} & \multicolumn{2}{l|}{$\begin{array}{l}\text { CPU- with two } \\
\text { cores }\end{array}$} & \multicolumn{2}{l|}{$\begin{array}{l}\text { CPU- with } \\
\text { three cores }\end{array}$} & \multicolumn{2}{l|}{$\begin{array}{l}\text { CPU- with four } \\
\text { cores }\end{array}$} & \multicolumn{2}{l|}{ GPU-GTX560TI } \\
\hline Time(s) & SUR & Time(s) & SUR & Time(s) & SUR & Time(s) & SUR & Time(s) & SUR \\
\hline 64.01 & 1 & 52.34 & 1.22 & 38.93 & 1.64 & 24.19 & 2.65 & $\mathbf{6 . 9 7}$ & $\mathbf{9 . 1 8}$ \\
\hline
\end{tabular}

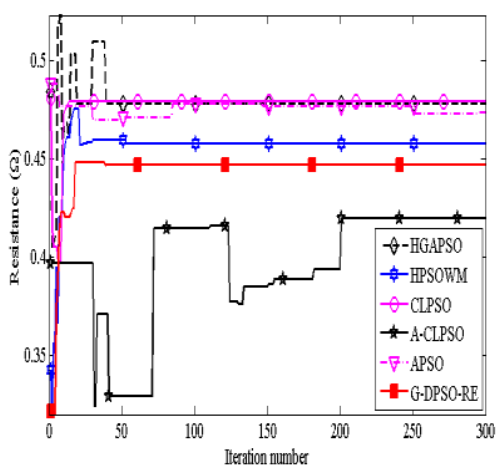

(a)

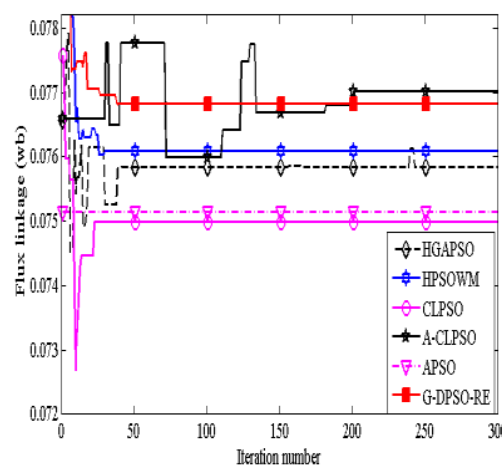

(b)

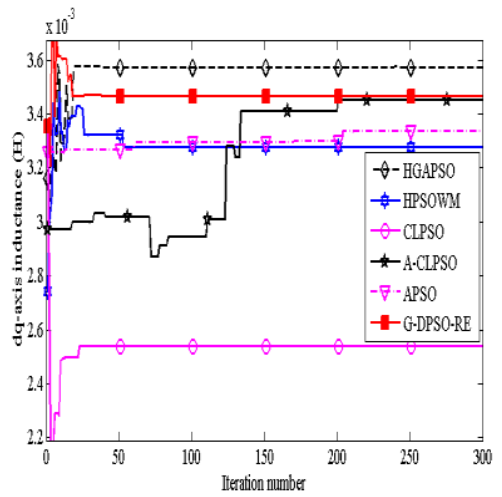

(c)

Fig. 11. Identified parameters under variation temperature condition (a) winding resistance. (b) rotor flux linkage (c) $d q$-axis inductance.

\section{Speedup Achieved by GPU Parallel Implementation}

The speed-up ratio is defined as $S U R=T \mathrm{~s} / T \mathrm{p}$, where $T s$ and $T p$ are the execution runs of the serial and parallel algorithms respectively. In this work speedup ratio is used to evaluate the efficiency of our proposed method implemented on different multi-core architecture. A GTX560TI GPU and multi-core CPU systems (range from one core to four cores) are compared in terms of computation speedup when applied to the estimation of PMSM parameters. The results are shown in
Table IV and Fig.12, where the symbol CPU-1, CPU-2, CPU-3, and CPU-4 are means CPU with one core, two cores, three cores, and four cores respectively.

The results show that the execution time of the G-DPSO-RE reduces greatly when it runs on increasing multi-core CPU. For example, it requires 64.01s, $52.34 \mathrm{~s}, 38.93 \mathrm{~s}$ and $24.19 \mathrm{~s}$ for running with one core, two cores, three cores and four cores CPU under the normal temperature condition, respectively. Whereas, the average time required for GPU is only 6.97s. The computation of 
optimal solution is accelerated by $9.18 \times$ in comparison of a sequential execution on CPU through exploiting the massively parallel architecture of GPUs. This fact shows that the speed and efficiency of the proposed parameter estimation method has been remarkably improved by GPU parallel execution. There are two main reasons behind this. Firstly, the computing speed of GPU with hundreds of threads is much faster than that of CPUs. Secondly, the proposed estimator involves mass intensive computing including data and program because of the G-DPSO-RE with intrinsic parallel character.

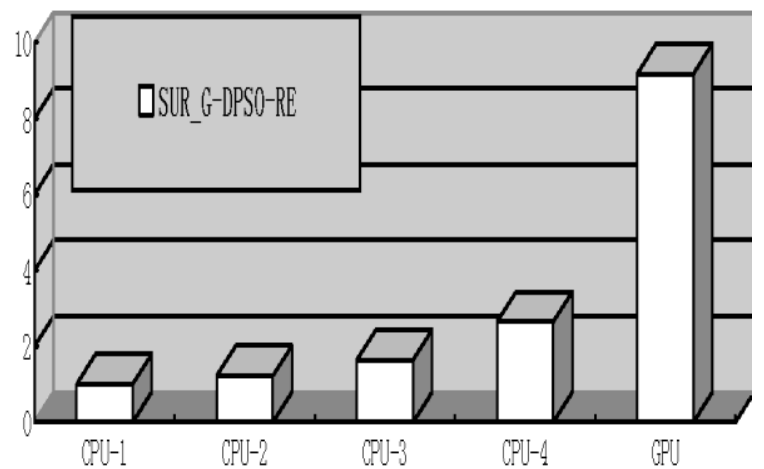

Fig.12. The comparison for SUR of G-DPSO-RE in PMSM parameter estimation parameter using different multi-core architecture using different multi-core architecture

\section{E Comparison Between With and Without Considering the VSI Nonlinearity}

In this section, an experimental is conducted to provide a comparison between the results with and without including the effect of VSI nonlinearity. Fig.13(a)-(c) show the estimated machine parameters (winding resistance, rotor flux linkage and $d q$-axis inductance) with and without considering the VSI nonlinearity under normal temperature condition using the proposed G-DPSO-RE. The estimated machine parameters without considering the VSI nonlinearity are as follows:: the estimated winding resistance $(R)$ is $0.453(\Omega)$, the estimated rotor flux linkage $\left(\psi_{m}\right)$ is $75.9(\mathrm{mWb})$ and the estimated $d q$-axis inductance $(L)$ is $3.433(\mathrm{mH})$, while the estimates with considering the VSI nonlinearity are: $0.371(\Omega)$, $78.36(\mathrm{mWb})$ and $3.474(\mathrm{mH})$, respectively. It is obvious that the estimated values with considering the VSI nonlinearity are different from that without considering the VSI nonlinearity, especially for the estimated winding resistance value and the estimated flux linkage. For example, the estimated winding resistance value $(0.453 \Omega)$ without considering VSI nonlinearity is much larger than that $(0.371 \Omega)$ with considering VSI nonlinearity (with an error of $0.453-0.371) / 0.371 \approx 22 \%$. The estimated rotor flux linkage value $(75.95 \mathrm{mWb})$, without considering VSI nonlinearity, is obviously smaller than $78.36(\mathrm{mWb})$ which was estimated with considering VSI nonlinearity, with an error of 78.36-75.95)/78.36 $\approx 3 \%$.

The differences between the parameter estimates are are mainly accounted for by effect of the VSI nonlinear disturbance voltage (i.e, $V_{\text {dead }} . D_{\mathrm{d}}$ and $V_{\text {dead }} . D_{\mathrm{q}}$ in (3a) and (3b)) which results in an increase in the estimated winding resistance. From the experiment, the distort voltage $V_{\text {dead }}$ is about $0.1 \mathrm{~V}$, the two terms $D q . V_{\text {dead }}$ ( about $0.4 \mathrm{~V}$ ) and $D d . V_{\text {dead }}$ ( about $0.2 \mathrm{~V}$ ) could introduce an error into the estimation of the PMSM parameters. This can be analyzed by using the typical electrical parameters in Table I and equation (3b). Note that $R i_{q}$ is about $1.3 \mathrm{~V}, \omega \psi_{m}$ is about $12 \mathrm{~V}$, and $D q . V_{\text {dead }} /$ $R i_{q} \approx 30 \%, D q . V_{\text {dead }} / \omega \psi_{m} \approx 3 \%$, so, after Clarke and Park transforms, the $d q$-axis voltage will change because $D q . V_{\text {dead }}$ can significantly affect the winding resistance and flux linkage estimation significantly.

The results show that the proposed parameter estimation model with considering the effect of VSI nonlinearity can improve the accuracy for machine parameter estimation.

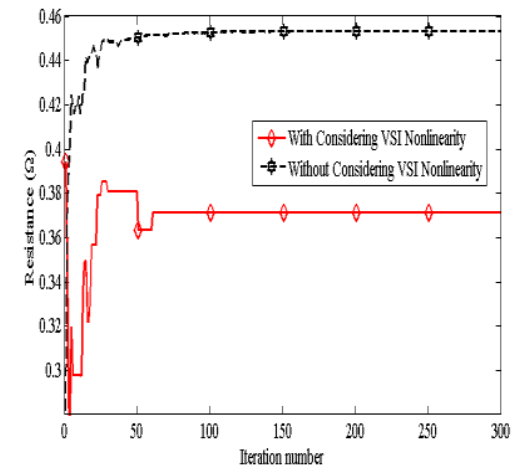

(a)

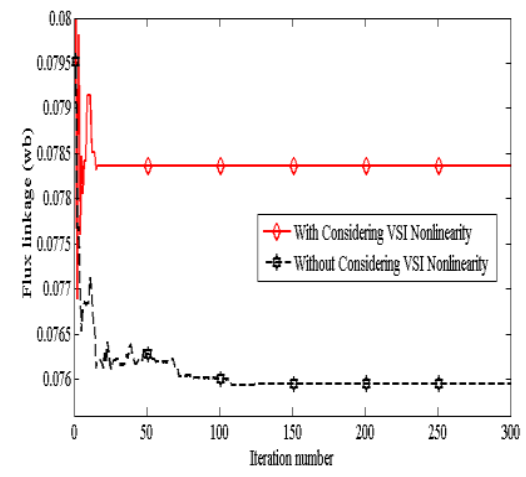

(b)

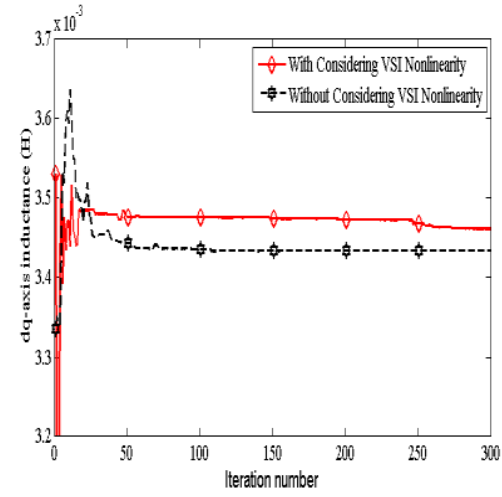

(c)

Fig13.The comparison of the identified parameters between with considering VSI nonlinearity and without considering VSI nonlinearity under normal temperature condition using the proposed G-DPSO-RE (a) winding resistance. (b) rotor flux linkage (c) $d q$-axis inductance

\section{CONCLUSION}

In this study, an accurate estimation model of combining the SPMSM parameters with VSI nonlinearity is established. A labor-division based dynamic PSO combined immune receptor editing strategy is designed for dynamic optimization and parallel implementation on GPU to accelerate the convergence process for parameter estimation. The computational efficiency of the parameter estimation procedure is greatly improved by the GPU parallel computing technique. The proposed parameter optimization method can be used to collectively estimate several parameters including 
the resistance, inductance, rotor flux along with VSI disturbance voltage with no expensive equipment. The influence of the VSI nonlinearity on the accuracy of the parameter estimation is also analyzed. In comparison with other PSO algorithms, the proposed estimation algorithm is relatively more complicated and the implementation of GPU techniques needs some special knowledge and programming skills, and this may be a major challenge for most of control or electrical engineers. But with the development of computer technology, it can be expected that this estimation algorithm can become easier for commercial PMSM drives. The proposed parameter estimation model focuses on steady state operation of machines and may not be suitable for other complex cases, for example for machine operating under time-varying operating conditions. This is a limitation of the proposed method. In our future work, we will investigate a new dynamic parameter estimation model for machine parameter estimation under dynamic operating state including the consideration of variation in the load disturbance and speed.

\section{References}

[1] W. Su, H. Eichi, W. Zeng, and M.-Y. Chow, "A survey on the electrificationof transportation in a smart grid environment," IEEE Trans. Ind.Informat., vol. 8, no. 1, pp. 1-10, Feb. 2012.

[2] G.L. Wang, L.Z. Qu, H.L. Zhan, J. Xu, L. Ding, G.Q Zhang, and D.G.Xu. Self-Commissioning of Permanent Magnet Synchronous Machine Drives at Standstill Considering Inverter Nonlinearities. IEEE Trans. Power Electron. vol.29, no. 12, pp.6615-6627, Dec, 2014

[3] S. Underwood, and I. Husain, "On-line parameter estimation and adaptive control of permanent magnet synchronous machines," IEEE Trans. Ind. Electron., vol. 57, no. 7, pp. 2435-2443, Jun. 2010.

[4] A. S. Babel,J. G. Cintron-Rivera, S. N. Foster,, and Strangas, E. G. Evaluation of a Parameter Identification Method for Permanent Magnet AC Machines Through Parametric Sensitivity Analysis. IEEE Trans. Energy Conver, vol.29,no.1,pp. 240-249, March,2014.

[5] N. Leboeuf et al., "Inductance calculations in permanent-magnet motors under fault conditions," IEEE Trans. Magn., vol. 48, no. 10, pp. 2605-2616, Oct. 2012.

[6] J. C. Uresty, J. R. Riba, and L. Romeral, "A back-emf based method to detect magnet failures in PMSMs," IEEE Trans. Magn., vol. 49, no. 1, pp. 591-598, Jan. 2013.

[7] Y. Park, S.K. Sul, "Sensorless Control Method for PMSM Based on Frequency-Adaptive Disturbance Observer, "IEEE Journal of Emerging and Selected Topics in Power Electronics,vol.2,no.2,pp.143-151,June,2014

[8] D. D. Reigosa, F. Briz, P. Gacia, J. M. Guerrero, and M.W. Degner, "Magnet temperature estimation in surface PM machines using high-frequency signal injection," IEEE Trans. Ind. Appl., vol. 46, no. 4, pp. 1468-1475,Aug. 2010

[9] YC Shi, K Sun, LP Huang, and Y. Li,"Online Identification of Permanent Magnet Flux.Based on Extended Kalman Filter for IPMSM Drive With Position Sensorless Control". IEEE Trans. Ind. Electron, vol.59,no.11,pp. 4169-4178, Nov ,2012.

[10] M Rashed, P F A Macconnell, A F Stronach, andP Acarnley. "Sensorless indirect-rotor-field-orientation speed control of a permanent-magnet synchronous motor with stator resistance estimation," IEEE Trans Ind. Electron.,vol.54, no.3,pp. 1664-1675, Mar.2007

[11] S. Kallio, J. Karttunen,, P. Peltoniemi,, P. Silventoinen, O. Pyrhonen, "Online Estimation of Double-Star IPM Machine Parameters Using RLS Algorithm", IEEE Trans. Ind. Electron., vol.61,no.9, pp. 4519 - 4530,Sept. 2014.
[12] M Hamida, J De Leon, A Glumineeau,and R Boisliveau "An Adaptive Interconnected Observer for Sensorles Control of PM Synchronous Motors With Online Parameter Identification" IEEE Trans. Ind. Electron., vol. 60, no. 2, pp. 739-748, Feb. 2013.

[13] H. W. Kim, M. J. Youn, K. Y. Cho, and H. S. Kim, "Nonlinearity estimation and compensation of PWM VSI for PMSM under resistance and flux linkage uncertainty," IEEE Trans. Control Syst. Technol., vol.14, no. 4,pp. 589-601, Jul. 2006.

[14] K H Kim, "Dual estimator-based compensation for dead time and nonlinearity unaffected by parameter variations in PMSM drive," Electronics letters, vol.48,no.23, pp. 1457-1459, Nov.2012.

[15] Z .H. Liu , X.H. Li ,H.Q. Zhang, L.H.Wu, and K. Liu. "An Enhanced Approach for Parameter Estimation Using Immune Dynamic Learning PSO Based on Multi-core Architecture," IEEE Syst., Man, Cybern Maga, vol. 2,no.1,pp.26-33,Jan.2016

[16] W.M. Lin,T.J. Su, and R.C.g Wu, "Parameter Identification of Induction Machine With a Starting No-Load Low-Voltage Test",IEEE Trans.Ind.Electron, vol. 59, no. 1, pp.352-360,Jan ,2012

[17] O. Sandre-Hernandez, R. Morales-Caporal, J. Rangel-Magdaleno, H. Peregrina-Barreto, and J. N. Hernandez-Perez, "Parameter identification of PMSMs using experimental measurements and a PSO algorithm," IEEE Trans. Instrum. Meas., vol.64,no.8,pp.2146-2154,Aug. 2015

[18]Z. H. Liu, J. Zhang, S. W. Zhou, X. H. Li, and K. Liu, “Coevolutionary particle swarm optimization using AIS and its application in multiparameter estimation of PMSM," IEEE Trans. Cybern., vol. 43, no. 6,pp. 1921-1935. Dec. 2013.

[19] Z. H. Liu, X. H. Li, L. H. Wu, S. W. Zhou, and K. Liu, "GPU-accelerated parallel coevolutionary algorithm for parameters identification and temperature monitoring in permanent magnet synchronous machines," IEEE Trans. Ind. Informat, vol. 11, no. 5, pp. 1220-1230, Oct. 2015.

[20] Z.H. Liu , H.L .Wei, Q.C . Zhong, and K. Liu Parameter Estimation for VSI-Fed PMSM based on a Dynamic PSO with Learning Strategies, IEEE Trans. Power Electron, vol. 32,no.4,pp.3154-3165 , April .2017

[21] V. Roberge, M. Tarbouchi, F. Okou, " Strategies to accelerate harmonic minimization in multilevel inverters using a parallel genetic algorithm on graphical processing unit," IEEE Trans.Power electron., vol.29,no.10,pp. 5087-5090,oct,2014.

[22]S. Morimoto, M. Sanada, and Y. Yakeda, "Mechanical sensorless drives of IPMSM with online parameter identification," IEEE Trans. Ind. Appl., vol. 42, no. 5, pp. 1241-1248, Sep./Oct. 2006.

[23] K. Liu, Z. Q. Zhu, Q. Zhang, and J. Zhang, "Influence of nonideal voltage measurement on parameter estimation in permanent magnet synchronous machines," IEEE Trans. Ind. Electron., vol. 59, no. 6, pp. 2438-2447, Jun.2012.

[24]C. Attaianese, V. Nardi, V.and G. Tomasso G, "Self-commissioning of induction motors fed by VSI", Electric Power Components and Systems Journal,vol.30,no.1,pp.1-9,Jan,2002.

[25]D. M. Gordon, "The organization of work in social insect colonies," Complexity, vol. 8, no. 1, pp. 43-46, 2002.

[26]M. Castillo-Cagigal, E.Matallanas, I.Navarro, E.C.Martín,F.M.Huelin and Á.Gutiérrez, "Variable Threshold Algorithm for Division of Labor Analyzed as a Dynamical System,"IEEE Trans.Cybern., vol. 44, no. 12, pp.2242-2252,Dec, 2014

[27] K .Vasileios. Karakasis, and A. Stafylopati, "Efficient Evolution of Accurate Classification Rules Using a Combination of Gene Expression Programming and Clonal Selection," IEEE Trans.Evolut.Comput., vol. 12, no. 6, pp.662-678,Dec,2008

[28] B. Zhou, K. W. Chan. Tao Yu, J. Tang, "Strength Pareto Multi-Group Search Optimizer for Multiobjective Optimal VAR Dispatch". IEEE Trans. 
Ind. Informat.,vol.2,no.10,pp.1012-1022,May,2014

[29]C. F. Juang, "A hybrid of genetic algorithm and particle swarm optimization for recurrent network design," IEEE Trans. Syst., Man, Cybern. B,Cybern, vol. 34, no. 2, pp. 997-1006, Apr. 2004.

[30] S.H .Ling, H.H.C .Iu; K.Y. Chan,H.K .Lam, B.C.W .Yeung and F.H. Leung," Hybrid Particle Swarm Optimization With Wavelet Mutation and Its Industrial Applications ", IEEE Trans. Syst., Man, Cybern. B,Cybern., vol.38,no.3, pp. 743 - 763, Jun. 2008.

[31]J. J. Liang, A. K. Qin, P. N. Suganthan, and S. Baskar, "Comprehensive learning particle swarm optimizer for global optimization of multimodal functions," IEEE Trans. Evol. Comput., vol. 10, no. 3, pp. 281-295,Jun. 2006.

[32]H. Wu.J.P Geng, R.H. Jin,J.Z. Qiu, W.Liu, J.Chen,S, Liu,” An Improved Comprehensive Learning Particle Swarm Optimization and Its Application to the Semiautomatic Design of Antennas," IEEE Trans.Antennas and Propagation.,vol.57, no.10,pp. 3018-3028, Oct. 2009.

[33] Z.H.Zhan, J. Zhang, Y. Li, and H. S.H. Chung. "Adaptive Particle Swarm Optimization," IEEE Trans. Syst., Man, Cybern. B,Cybern., vol. 39, no. 6, pp.1362-1381.Dec.2009

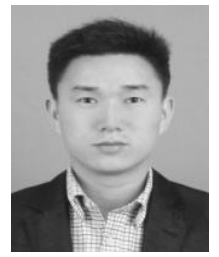

Zhao-Hua Liu (M'16) He received M.Sc. degree in computer science and engineering, and the $\mathrm{PhD}$. degree in automatic control and electrical engineering from the Hunan University, China, in 2010 and2012,respectively. He worked as a visiting researcher in the Department of Automatic Control and Systems Engineering at the University of Sheffield, United Kingdom, from 2015 to 2016.

$\mathrm{He}$ is currently an Associate Professor with the School of Information and Electrical Engineering, Hunan University of Science and Technology, Xiangtan, China. His current research interests include computational intelligence and learning algorithms design, parameter estimation and control of permanent-magnet synchronous machine drives, and condition monitoring and fault diagnosis for power system.

Dr. Liu has published a monograph in the field of Biological immune system inspired hybrid intelligent algorithm and its applications, and published more than 30 research papers in refereed journals and conferences, including IEEE TRANSACTIONS/JOURNAL/MAGAZINE .He is a regular reviewer for several international journals and conferences.

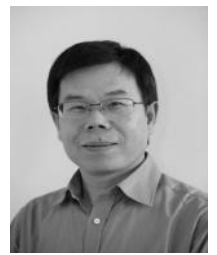

Hua-Liang Wei received the Ph.D. degrees in automatic control from The University of Sheffield, Sheffield, U.K., in 2004.

$\mathrm{He}$ is currently a senior lecturer with the Department of Automatic Control and Systems Engineering, The University of Sheffield, Sheffield, UK. His research focuses on evolutionary algorithms, identification and modelling for complex nonlinear systems, applications and developments of signal processing, system identification and data modelling to control engineering.

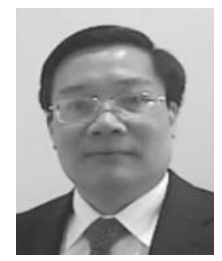

Qing-Chang Zhong (M'03-SM'04-F'17) received the Ph.D. degree in control and engineering from Shanghai Jiao Tong University, Shanghai, China, in 2000 and the Ph.D. degree in control and power engineering from Imperial College London, London, U.K., in 2004.

$\mathrm{He}$ is a Distinguished Lecturer of the IEEE Power Electronics Society and holds the Max McGraw Endowed Chair Professor in Energy and Power Engineering with the Department of Electrical and Computer Engineering, Illinois Institute of Technology, Chicago, IL, USA. the Chair Professor in Control and Systems Engineering with the Department of Automatic Control and Systems Engineering, The University of Sheffield, Sheffield, U.K. He (co)authored three research monographs, including Control of Power Inverters in Renewable Energy and Smart Grid Integration (Wiley-IEEE Press, 2013). His research focuses on power electronics and advanced control theory, together with their applications in various sectors. Dr. Zhong serves as an Associate Editor for the IEEE TRANSACTIONS ON AUTOMATIC
CONTROL, IEEE TRANSACTIONS ON POWER ELECTRONICS, IEEE TRANSACTIONS ON INDUSTRIAL ELECTRONICS, IEEE TRANSACTIONS ON CONTROL SYSTEMS TECHNOLOGY, IEEE ACCESS, IEEE JOURNAL OF EMERGING AND SELECTED TOPICS IN POWER ELECTRONICS.

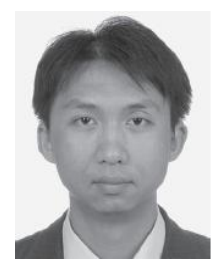

Kan Liu (M'14) received the B.Eng. and Ph.D. degrees in automation from Hunan University, Changsha,China, in 2005 and 2011 respectively. and the Ph.D. degree in electronic and electrical engineering from University of Sheffield, Sheffield, U.K., in 2013.

$\mathrm{He}$ is currently a Lecturer with the Control Systems Research Group, Loughborough University. His research interest focuses on parameter estimation and sensorless control of permanent-magnet synchronous machine drives and compensation of VSI nonlinearity.

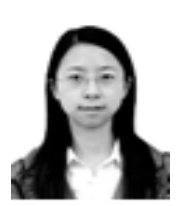

Xiao-Hua Li received the B.Eng. degree in computer science and technology from Hunan University of Science and Engineering, Yong zhou, China, in 2007 and the M.Sc. degree in computer science from Hunan University, Changsha, China, in 2010.Currently, She is currently a lecturer in the School of Information and Electrical Engineering, Hunan University of Science and Technology, Xiangtan, China. Her interests are in evolutionary computation. 